\title{
Influencia del riego sobre la composición y características organolépticas del aceite de oliva
}

\author{
Por J. Salas ${ }^{*}$, M. Pastor ${ }^{\star *}$, J. Castro** y V. Vega ${ }^{\star *}$ \\ *Laboratorio Agroalimentario de Córdoba. Consejería de Agricultura y Pesca. Apdo. 3045 - 14080 Córdoba \\ *Dpto. Olivicultura y Arboricultura Frutal. C.I.F.A. «Alameda del Obispo» Córdoba. \\ Consejería de Agricultura y Pesca. Apdo. 4240 - 14080 Córdoba
}

\section{RESUMEN}

Influencia del riego sobre la composición y características organolépticas del aceite de oliva.

Se estudia la composición relativa en ácidos grasos, características organolépticas y parámetros de calidad de los aceites producidos en olivares de secano o regados con diferentes dosis de agua. La composición acídica fue alterada por el riego. El contenido en ácidos grasos monoinsaturados y poliinsaturados fue mayor en los aceites de secano, mientras que el contenido relativo en ácidos grasos saturados fue mayor en regadío. Las relaciones insaturados/saturados y oleico/linoleico diferencian los aceites de riego y de secano. Existió una relación entre los valores de $\mathrm{K}_{225}$, el contenido en polifenoles y el grado de amargor, siendo los aceites de secano mucho más amargos que los de riego, discriminando estos tres parámetros los aceites de riego y los de secano. El grado de amargor disminuyó al aumentar la cantidad de agua aportada con el riego.

PALABRAS-CLAVE: Aceite de oliva virgen - Ácido graso Amargor - Calidad - Olivo - Riego.

\section{SUMMARY}

Irrigation effects on fatty acidic composition, organoleptic characteristics and other quality parameters of virgin olive oils.

Fatty acidic composition, organoleptic characteristics and other quality parameters of virgin olive oils were evaluated in dry-farming and irrigated olive orchards. The acidic composition was affected by irrigation. The content in monoinsaturated and poliinsaturated fatty acid was higher in the oils of the dry-farming olive orchard, while the relative content in saturated fatty acids was higher in the irrigated one. The insaturated/saturated and oleic/linoleic relations distinguish the irrigated and non irrigated olive trees. The $\mathrm{K}_{225}$ values, the poliphenolic contents and the bitter taste were also correlated. The oils from non irrigated olive trees showed the higher bitter taste. Those three parameters discriminated the oils from irrigated and non irrigated trees. The bitter taste diminished when increasing the amount of water supplied with the irrigation.

KEY-WORDS: Bitter taste - Fatty acid - Irrigation - Olive tree - Quality - Virgin olive oil.

\section{INTRODUCCIÓN}

En la moderna olivicultura la obtención de aceites de calidad debe ser también uno de los objetivos prioritarios del agricultor, ya que el mercado podría y debería pagar los aceites en función de la calidad de los mismos. Además de una inadecuada manipulación de las aceitunas durante la recepción en fábrica y en el proceso de extracción, que pueden alterar negativamente un producto que suele venir del campo con una calidad bastante satisfactoria, otros factores como la variedad (Uceda y Hermoso, 1994), época de recolección, medio de producción y técnicas de cultivo, pueden afectar a la composición y características organolépticas de los aceites (Civantos et al., 1992; Humanes y Civantos, 1993).

En la provincia de Jaén es cada vez mayor la superficie de olivar regado, pudiéndose decir que más de ochenta mil hectáreas reciben aportaciones de agua en algún momento de su ciclo vegetativo. El riego aumenta la cantidad de aceite producido por el olivo (Michelakis, 1995), efecto que es aún más marcado en los años de baja pluviometría (Pastor y Orgaz, 1994). La influencia del riego sobre la composición y calidad del aceite de oliva no está aún suficientemente estudiada. Recientes trabajos realizados en Italia (Rotundo et al., 1993; Dettori y Russo, 1993; Inglese et al., 1996) han estudiado la composición de los aceites producidos en olivos de riego y en secano, sin haber encontrado claras diferencias, probablemente porque estos autores trabajaron en zonas y años con alta pluviometría, por lo que los árboles de secano no llegaron a padecer un marcado déficit hídrico. Beltrán et al., (1995) observaron en años secos cómo aceites de olivos de la variedad Arbequina cultivados en secano en Córdoba presentaban un mayor contenido en polifenoles totales, así como un mayor $\mathrm{K}_{225}$ y estabilidad que el de los olivos cultivados en regadío.

En el presente trabajo se estudia la composición acídica, características organolépticas y parámetros de calidad reglamentada de los aceites producidos durante tres años en secano y bajo diferentes regíme- 
nes de riego, en la comarca de El Condado en la provincia de Jaén. Los resultados obtenidos indican que se registran cambios en los parámetros estudiados y que éstos podrían atribuirse a los diferentes aportes hídricos realizados.

\section{MATERIAL Y MÉTODOS}

El estudio se ha realizado durante los años 1993, 1994 y 1995 en un olivar tradicional adulto centenario en la variedad Picual en la finca Los Robledos en el t.m. de Santisteban del Puerto (Jaén), plantado con una densidad de 80 olivos/ha. Los árboles se riegan con agua procedente de un pozo profundo del que se extrae un agua de bastante buena calidad (CE $=0,85$ $\mathrm{dS} / \mathrm{m}$ ). Los regímenes de riego empleados fueron los siguientes: a) programa siguiendo la metodología FAO (Doorembos y Pruit, 1977), habiéndose aportado anualmente $3.000 \mathrm{~m} 3 / \mathrm{ha}$, cubriendo las necesidades teóricas de la planta para el año de climatología media; b) riego durante el período 1 de abril a 31 de octubre aplicando diariamente $120 \mathrm{~K} /$ olivo, equivalente a una aportación anual de $2.000 \mathrm{~m}^{3} / \mathrm{ha}$; c) riego en el período 1 de marzo a 31 de octubre aplicando diariamente 80 //olivo, equivalente a una aportación anual de $1.500 \mathrm{~m}^{3} / \mathrm{ha}$; d) control no regado (secano). La figura 1 muestra las aportaciones mensuales de agua de riego en cada uno de los tratamientos. Se empleó un diseño experimental en bloques al azar, con 4 repeticiones por tratamiento y parcelas elementales de 12 olivos.
En los años 1993, 1994 y 1995 en los que se ha realizado este estudio, las pluviometrías medias fueron muy inferiores a la media, 380,320 y $420 \mathrm{~mm}$ respectivamente, con lluvias en este último año concentradas en los meses de junio (tormenta) y en noviembre y diciembre, cuando la cosecha ya estaba decidida. Debido a la sequía padecida en 1993 y 1994, años de carga en regadío (Pastor y Orgaz, 1994; Pastor et al., 1996), el riego proporcionó unos importantísimos aumentos de producción con respecto al secano (datos no presentados), mientras que en 1995 las producciones fueron bastante bajas en todos los tratamientos, especialmente en secano, en donde sólo hubo producción en una de las cuatro repeticiones del ensayo.

Las aceitunas fueron recolectadas casi siempre del árbol en todos los tratamientos y en estado de madurez comprendido entre 4 y 4,5 (epidermis color negro sin penetrar esta coloración en la pulpa), si bien en el último año gran parte de los frutos del tratamiento $F A O$ ya habían caído al suelo cuando se realizó la recolección. Los aceites fueron extraídos en laboratorio empleando el método Abencor, batiendo la pasta, una vez molturadas las aceitunas, a una temperatura de $28^{\circ} \mathrm{C}$ durante 40 minutos, añadiéndose $30 \mathrm{~g}$ de microtalco por cada kilogramo de frutos, y agua tibia 10 minutos antes del final del proceso de batido. Los aceites obtenidos fueron conservados en botellas oscuras cerradas herméticamente y congelados, para evitar alteraciones, hasta que fueron utilizadas para las determinaciones analíticas correspondientes.

Las determinaciones realizadas fueron las siguientes: acidez libre (norma UNE 55.011), índice de peróxidos (norma UNE 55.023), absorbancia al ultravioleta

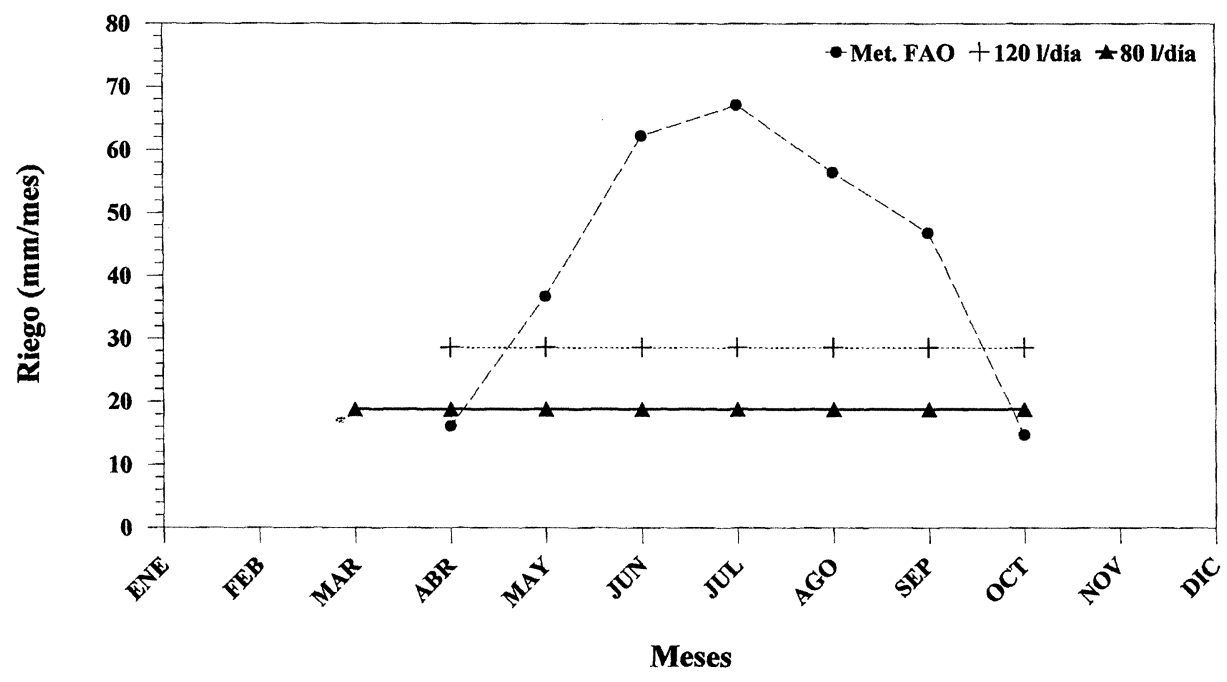

Figura 1

Programas de riego aplicados 
$\left(\mathrm{K}_{270}\right.$ y $\left.\mathrm{K}_{332}\right)$ (norma UNE 55.047-73), composición acídica (norma UNE 55.037), contenido en polifenoles y $\mathrm{K}_{225}$ (Gutiérrez Rosales et al., 1992) y, estabilidad oxidativa (medida en Rancimat $100^{\circ} \mathrm{C}$, norma UNE 55.166). La valoración organoléptica de los aceites (panel analítico) fue realizada por el panel del Laboratorio Agroalimentario de Córdoba compuesto por 12 personas, que igualmente evaluó diferentes atributos del aceite, tales como frutado, amargo y picante. Una vez catalogados los aceites, se pidió también al panel que ordenara las muestras, de cada uno de los cuatro tratamientos, de mayor a menor grado de amargor.

Tratando de evaluar de una forma objetiva el estado hídrico de los árboles, periódicamente se midió el potencial de agua en hoja antes del amanecer, empleando para ello una cámara de presión y utilizando brotes del crecimiento del año con dos pares de hojas. Este parámetro puede indicarnos el grado de estrés hídrico al que estamos sometiendo a los árboles (Dettori, 1987; Goldhamer et al.,1994).

El análisis estadístico de los resultados se ha realizado mediante análisis de varianza, separando las medias aplicando el test de la mínima diferencia significativa.

\section{RESULTADOS}

La figura 2 muestra la evolución de los potenciales de agua en hoja antes del amanecer durante el período 22/9/94-16/11/95. Si tenemos en cuenta que diversos autores (Dettori, 1987; Goldhamer et al., 1994) consideran que el valor umbral de estrés hídrico para el olivo podría situarse en torno a $-0.5 \mathrm{MPa}$, observamos cómo se provocó déficit hídrico en todos los tratamientos, lo cual puede explicarse en el tratamiento teóricamente mejor regado (Met. FAO) por el hecho de que, tal como estaba previsto en el protocolo del ensayo, se aplicó en estos años un programa de riego que optimizaría las necesidades de agua en un año de pluviometría media, mientras que en los años en los que se realizó este estudio la pluviometría observada fue muy inferior a la media. En la citada figura podemos igualmente observar como en el tratamiento con menor dotación de riego $(80 \mathrm{l} / \mathrm{ol})$ y en secano, en determinadas épocas del año se observaron valores del potencial hídrico significativamente menores (árboles con mayor estrés hídrico) que los de los dos tratamientos mejor regados (120 l/ol y Met. FAO), siendo mayores estas diferencias durante el verano y otoño (hasta las primeras lluvias), época en la que se produce el crecimiento de los frutos, así como la formación del aceite, procesos a los que igualmente afectó el régimen hídrico aplicado (Pastor et al., 1996).

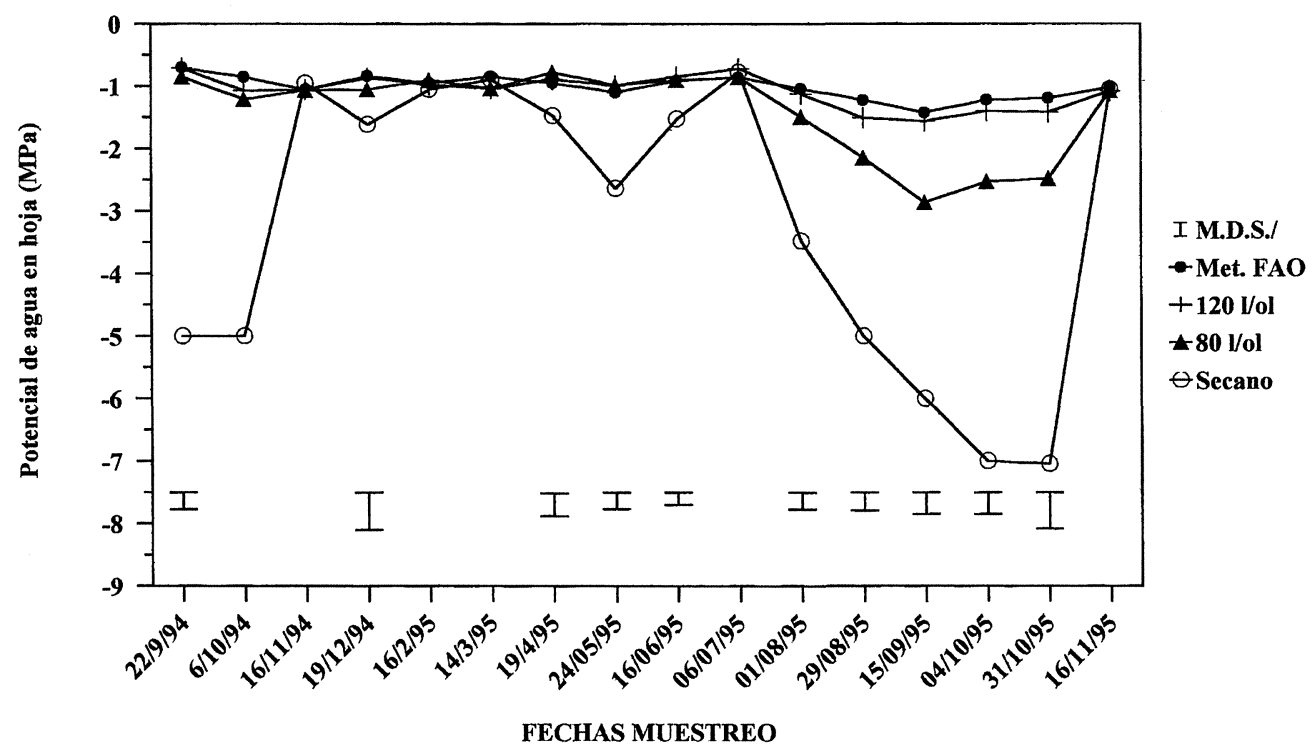

Figura 2

Evolución del potencial de agua en hoja en medición realizada antes del amanecer, en los diferentes tratamientos de riego 
La tabla I muestra la composición en ácidos grasos de los aceites en los años 1993, 1994 y 1995 para los diferentes tratamientos de riego. En los años 1993 y 1994, los resultados fueron bastante similares, observándose en todos los casos una composición típica en los aceites de la variedad Picual (Frías et al., 1991), mientras que en el año 1995 la composición acídica no presentó la composición típica de los aceites de esta variedad, observándose contenidos en ácido oleico próximos al $70 \%$ y un anormalmente alto contenido en ácidos palmítico y linoleico. Como se dijo anteriormente, en este año la producción de los olivos fue bastante baja, especialmente en secano, produciéndose un crecimiento exagerado de los frutos, que en los árboles regados llegaron a alcanzar un tamaño medio entre 8 y 10 gramos/aceituna (datos no presentados), anormalmente grandes para esta variedad, razón a la que podríamos atribuir probablemente las anormalidades observadas, por lo que este año no debería tenerse muy en cuenta para el análisis de alguno de los resultados obtenidos.

En los años 1993 y 1994, en los que la diferencia de producción entre los olivos de riego y los de secano fue muy grande, se observan diferencias significativas en la composición relativa de ácidos grasos (Tabla I) debidas en parte a la cantidad de agua de riego aplicada, y también, posiblemente, debido a las propias diferencias de producción existente entre riego y secano. El contenido en ácidos grasos monoinsaturados (Tabla II) y en especial en ácido oleico (Tabla I) fue mayor en secano y en los tratamientos con menor cantidad de agua de riego, observándose igualmente en secano un mayor contenido en ácidos grasos poliinsaturados (linoleico y linolénico). Por el contrario, los aceites de riego presentaron una mayor proporción de ácidos grasos saturados (palmítico y esteárico). La relación insaturados/saturados (Tabla II) fue significativamente menor en los dos tratamientos mejor regados, mientras que la relación monoinsaturados/poliinsaturados y la relación oleico/linoleico permitió diferenciar en este ensayo los aceites de riego y secano, sin que se observaran diferencias significativas entre las diferentes dosis de riego. Sería interesante comprobar estos aspectos en años en que las diferencias de producción entre riego y secano no fuesen tan importantes.

La Tabla III muestra los valores de diferentes parámetros analíticos de calidad reglamentada (acidez, índice de peróxidos, $K_{270}$ y $K_{232}$ ). Los valores de absorbancia al UV $\left(K_{270}\right)$, parámetro que estima el estado de oxidación y conservación de un aceite, fueron significativamente más altos en los aceites de secano, estando todos los tratamientos, en los tres años, dentro de los límites reglamentados para que puedan ser considerados como vírgenes de calidad extra. Estos resultados parecen estar de acuerdo con las diferentes coloraciones y tonalidades observadas en los aceites (datos no cuantificados). Mientras que los aceites de secano presentaban colores verdosos, los de riego

Tabla 1

Composición relativa en ácidos grasos de los aceites producidos en los años 1993, 1994 y 1995 en los diferentes programas de riego

\begin{tabular}{|c|c|c|c|c|c|c|c|c|c|}
\hline \multirow{2}{*}{ AÑO } & \multirow{2}{*}{$\begin{array}{c}\text { TRATAMIENTOS } \\
\text { DE RIEGO } \\
\text { Met. FAO }\end{array}$} & \multirow[b]{2}{*}{$\begin{array}{c}\text { Palmitoleico } \\
\text { C16-1 }\end{array}$} & \multicolumn{7}{|c|}{ ÁCIDOS GRASOS \% } \\
\hline & & & \multicolumn{2}{|c|}{$\begin{array}{c}\text { Palmítico } \\
\text { C16 }\end{array}$} & $\begin{array}{c}\begin{array}{c}\text { Esteárico } \\
\text { C18 }\end{array} \\
3,28 \mathrm{a}\end{array}$ & \begin{tabular}{|l|} 
Oléico \\
C18-1
\end{tabular} & $\begin{array}{c}\begin{array}{c}\text { Linolénico } \\
\text { C18-3 }\end{array} \\
0,63 \mathrm{a}\end{array}$ & $\begin{array}{c}\begin{array}{c}\text { Aráquico } \\
\text { C20 }\end{array} \\
0,30 \mathrm{a}\end{array}$ & $\begin{array}{c}\begin{array}{c}\text { Gadoleico } \\
\text { C20-1 }\end{array} \\
0,25 \mathrm{a}\end{array}$ \\
\hline 1993 & $\begin{array}{l}\text { Met. FAO } \\
120 \text { l/día } \\
80 \text { l/día } \\
\text { Secano }\end{array}$ & $\begin{array}{l}0,95 \mathrm{a} \\
0,83 \mathrm{ab} \\
0,83 \mathrm{ab} \\
0,75 \mathrm{~b}\end{array}$ & $\begin{array}{l}10,3 \\
10 \\
9,38 \\
9,33\end{array}$ & $\begin{array}{l}a \\
a \\
b \\
b\end{array}$ & $\begin{array}{ll}3,28 & a \\
3,4 & a \\
3,28 & a \\
2,83 & b\end{array}$ & $\begin{array}{r}80,55 \text { a } \\
80,88 \text { a } \\
1,66 \text { b } \\
81,70 \text { b }\end{array}$ & $\begin{array}{l}0,63 \mathrm{a} \\
0,63 \mathrm{a} \\
0,63 \mathrm{a} \\
0,68 \mathrm{a}\end{array}$ & $\begin{array}{l}0,30 \mathrm{a} \\
0,30 \mathrm{a} \\
0,28 \mathrm{a} \\
0,33 \mathrm{a}\end{array}$ & $\begin{array}{l}0,25 \mathrm{a} \\
0,35 \mathrm{a} \\
0,25 \mathrm{a} \\
0,23 \mathrm{a}\end{array}$ \\
\hline 1994 & $\begin{array}{l}\text { Met. FAO } \\
120 \mathrm{l} / \text { día } \\
80 \mathrm{I} / \text { día } \\
\text { Secano }\end{array}$ & $\begin{array}{l}1,13 \text { a } \\
0,93 \text { a } \\
0,98 \text { a } \\
1,03 \text { a }\end{array}$ & $\begin{array}{l}11,1 \\
10 \\
10,7 \\
9,78\end{array}$ & $\begin{array}{l}\mathrm{b} \\
\mathrm{ab} \\
\mathrm{b} \\
\mathrm{a}\end{array}$ & $\begin{array}{ll}3,53 & a \\
3,53 & a \\
3,71 & a \\
2,23 & b\end{array}$ & $\begin{array}{l}78,38 \mathrm{a} \\
80,35 \mathrm{~b} \\
79,55 \mathrm{ab} \\
80,05 \mathrm{~b}\end{array}$ & $\begin{array}{l}0,75 \mathrm{~b} \\
0,61 \mathrm{a} \\
0,53 \mathrm{a} \\
0,88 \mathrm{~b}\end{array}$ & $\begin{array}{l}0,30 \mathrm{~b} \\
0,30 \mathrm{~b} \\
0,33 \mathrm{~b} \\
0,25 \mathrm{a}\end{array}$ & $\begin{array}{l}0,28 \mathrm{a} \\
0,25 \mathrm{a} \\
0,23 \mathrm{a} \\
0,31 \mathrm{a}\end{array}$ \\
\hline 1995 & $\begin{array}{l}\text { Met. FAO } \\
120 \text { l/día } \\
80 \text { l/día } \\
\text { Secano }\end{array}$ & $\begin{array}{ll}1,91 & c \\
1,55 & b \\
1,43 & b \\
1,11 & a\end{array}$ & $\begin{array}{l}13,6 \\
12,9 \\
12,9 \\
13,1\end{array}$ & $\begin{array}{l}\mathrm{b} \\
\mathrm{a} \\
\mathrm{a} \\
\mathrm{a}\end{array}$ & $\begin{array}{l}4,35 \mathrm{~b} \\
4,55 \mathrm{~b} \\
4,35 \mathrm{~b} \\
3,81 \mathrm{a}\end{array}$ & $\begin{array}{l}67,53 \text { a } \\
70,03 \text { a } \\
70,41 \text { a } \\
70,71 \text { a }\end{array}$ & $\begin{array}{l}0,90 c \\
0,75 a b \\
0,70 a \\
0,80 b\end{array}$ & $\begin{array}{l}0,25 \mathrm{a} \\
0,30 \mathrm{a} \\
0,30 \mathrm{a} \\
0,30 \mathrm{a}\end{array}$ & $\begin{array}{l}0,20 \mathrm{a} \\
0,20 \mathrm{a} \\
0,20 \mathrm{a} \\
0,20 \mathrm{a}\end{array}$ \\
\hline
\end{tabular}

En cada año, los valores de cada columna seguidos por letras diferentes difieren significativamente al nivel $p \leq 0,05$ según el test de la M.D.S. 
Tabla II

Composición relativa en ácidos grasos de los aceites producidos en los años 1993, 1994 y 1995 en los diferentes programas de riego

\begin{tabular}{|c|c|c|c|c|c|c|c|c|}
\hline \multirow[b]{2}{*}{ AÑO } & \multirow[b]{2}{*}{$\begin{array}{l}\text { TRATAMIENTOS } \\
\text { DE RIEGO }\end{array}$} & \multicolumn{4}{|c|}{ ÁCIDOS GRASOS (\%) } & \multicolumn{3}{|c|}{ RELACIONES } \\
\hline & & Poliinsatur. & Monoinsat. & Insaturados & Saturados & $\begin{array}{c}\text { Oleico/ } \\
\text { Linoleico }\end{array}$ & $\begin{array}{l}\text { Insaturado/ } \\
\text { Saturado }\end{array}$ & $\begin{array}{c}\text { Monoinsa/ } \\
\text { Poliinsat. }\end{array}$ \\
\hline 1993 & $\begin{array}{l}\text { Met. FAO } \\
120 \text { l/día } \\
80 \text { l/día } \\
\text { Secano }\end{array}$ & $\begin{array}{ll}4,15 & b \\
3,95 & c \\
4,05 & b c \\
4,65 & a\end{array}$ & $\begin{array}{ll}81,85 & b \\
82,15 & b \\
82,85 & a \\
82,77 & a\end{array}$ & $\begin{array}{l}86,01 \mathrm{c} \\
86,09 \mathrm{c} \\
86,89 \mathrm{~b} \\
87,43 \mathrm{a}\end{array}$ & $\begin{array}{r}14,03 \mathrm{a} \\
13,93 \mathrm{a} \\
13,11 \mathrm{~b} \\
1,66 \mathrm{~b}\end{array}$ & $\begin{array}{ll}22,86 & a \\
24,44 & a \\
24,01 & a \\
20,67 & b\end{array}$ & $\begin{array}{l}6,13 \mathrm{~b} \\
6,19 \mathrm{~b} \\
6,64 \mathrm{a} \\
6,91 \mathrm{a}\end{array}$ & $\begin{array}{l}19,73 \text { a } \\
20,89 \text { a } \\
20,57 \text { a } \\
17,83 \text { b }\end{array}$ \\
\hline 1994 & $\begin{array}{l}\text { Met. FAO } \\
120 \text { I/día } \\
80 \text { l/día } \\
\text { Secano }\end{array}$ & $\begin{array}{ll}4,95 & b \\
4,31 & b \\
4,51 & b \\
6,03 & a\end{array}$ & $\begin{array}{ll}79,88 & b \\
80,85 & a \\
81,63 & a \\
81,48 & a\end{array}$ & $\begin{array}{l}84,83 \mathrm{c} \\
85,15 \mathrm{c} \\
86,13 \mathrm{~b} \\
87,49 \mathrm{a}\end{array}$ & $\begin{array}{l}15,13 \mathrm{a} \\
14,88 \mathrm{ab} \\
14,03 \mathrm{~b} \\
12,45 \mathrm{c}\end{array}$ & $\begin{array}{ll}19,15 & a b \\
21,48 & a \\
20,61 & a \\
15,63 & b\end{array}$ & $\begin{array}{l}5,63 \mathrm{c} \\
5,75 \mathrm{bc} \\
6,15 \mathrm{~b} \\
7,03 \mathrm{a}\end{array}$ & $\begin{array}{l}16,49 \mathrm{ab} \\
19,23 \mathrm{a} \\
18,15 \mathrm{a} \\
13,58 \mathrm{~b}\end{array}$ \\
\hline 1995 & $\begin{array}{l}\text { Met. FAO } \\
120 \text { I/día } \\
80 \text { I/día } \\
\text { Secano }\end{array}$ & $\begin{array}{l}11,95 a \\
10,33 a \\
10,23 a \\
10,40 a\end{array}$ & $\begin{array}{l}69,73 \text { a } \\
71,87 \text { a } \\
72,12 \text { a } \\
72,10 \text { a }\end{array}$ & $\begin{array}{l}81,68 \text { a } \\
82,20 \text { a } \\
82,35 \text { a } \\
82,50 \text { a }\end{array}$ & $\begin{array}{l}18,26 \text { a } \\
17,76 \text { a } \\
17,64 \text { a } \\
17,30 \text { a }\end{array}$ & $\begin{array}{l}6,24 \text { a } \\
7,40 \text { a } \\
7,46 \text { a } \\
7,36 \text { a }\end{array}$ & $\begin{array}{l}4,48 \text { a } \\
4,63 \text { a } \\
4,68 \text { a } \\
4,80 \text { a }\end{array}$ & $\begin{array}{l}5,94 \text { a } \\
7,03 \text { a } \\
7,12 \text { a } \\
6,93 \text { a }\end{array}$ \\
\hline
\end{tabular}

En cada año, los valores de cada columna seguidos por letras diferentes difieren significativamente al nivel $p \leq 0,05$ según el test de la M.D.S.

presentaban color amarillo, correspondiendo las tonalidades menos intensas a los que procedían de los árboles mejor regados. El valor del $K_{232}$ fue en todos los años significativamente mayor en el aceite producido en secano.

Tabla III

Parámetros analíticos de calidad de los aceites producidos en los años 1993, 1994 y 1995

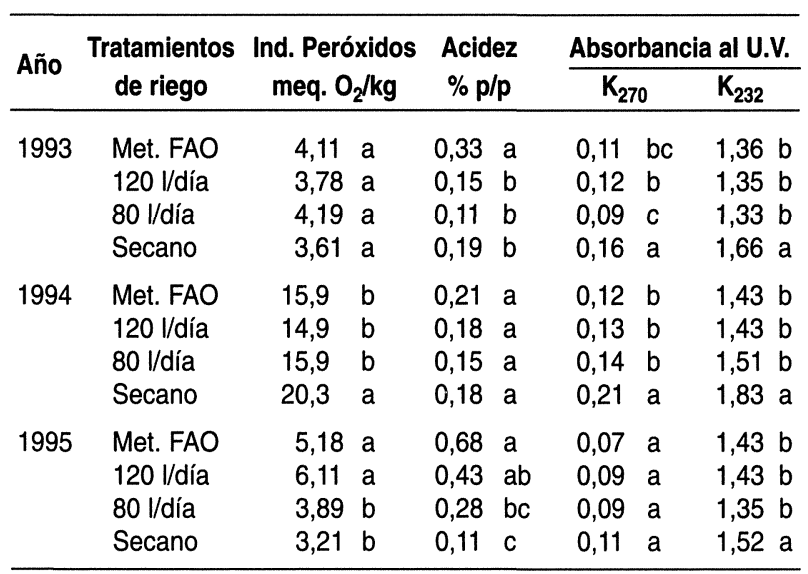

En cada año, los valores de cada columna seguidos por letras diferentes difieren significativamente al nivel $p \leq 0,05$ según el test de la M.D.S.
La acidez (Tabla III) fue significativamente mayor (años 1993 y 1995) en los aceites producidos en los árboles mejor regados (Met FAO). Una posible explicación podría ser el significativo mayor contenido en agua en los frutos a lo largo del proceso de crecimiento y maduración de las aceitunas en los árboles regados (Pastor et al., 1996), lo que podría favorecer los procesos de hidrólisis de los triglicéridos, y por tanto el aumento de la acidez del aceite. Sin embargo, estos resultados tienen una importancia relativa escasa, ya que la acidez observada en los aceites de todos los tratamientos está también dentro de los límites exigidos para que puedan ser considerados como vírgenes de calidad extra.

El índice de peróxidos valora el estado de oxidación inicial del aceite. En 1993 no se observan diferencias significativas en los índices de peróxidos de los aceites correspondientes a los diferentes tratamientos. Sin embargo, en 1994, debido probablemente a las heladas de final del mes de diciembre, pocos días antes de la recolección, el índice de peróxidos presentaba en todos los tratamientos valores anormalmente altos, significativamente menor en riego que en secano, superándose en este tratamiento el límite reglamentado, no observándose en dicho año diferencias significativas entre los distintos tratamientos de riego. Este hecho parece sugerir una menor susceptibilidad al frío de las aceitunas de los árboles de riego. En 1995 los resultados son diferentes a los del año anterior, observándose índices de peróxidos significativamente mayores en los dos tratamientos mejor regados. 
El contenido en polifenoles del aceite es un parámetro que en este ensayo nos permite diferenciar los aceites producidos en riego y en secano (Tabla IV), en especial en el año 1995, en el que el contenido en polifenoles aumentó significativamente al reducirse la cantidad de agua de riego aportada.

\section{Tabla IV}

Parámetros analíticos relacionados con el amargor de los aceites producidos en los años 1993, 1994 y 1995

\begin{tabular}{|c|c|c|c|c|c|}
\hline Año & $\begin{array}{l}\text { Tratamientos } \\
\text { de riego }\end{array}$ & $\begin{array}{c}\text { Polifenoles } \\
\text { (ac. cafeico, ppm) }\end{array}$ & $K_{225}$ & $\begin{array}{l}\text { Valoración } \\
\text { sensorial } \\
\text { amargor (1) }\end{array}$ & $\begin{array}{c}\text { Estabilidad } \\
\text { horas }\end{array}$ \\
\hline 1993 & $\begin{array}{l}\text { Met. FAO } \\
120 \text { l/día } \\
80 \text { l/día } \\
\text { Secano }\end{array}$ & $\begin{array}{l}\mathrm{s} / \mathrm{d} \\
\mathrm{s} / \mathrm{d} \\
\mathrm{s} / \mathrm{d} \\
\mathrm{s} / \mathrm{d}\end{array}$ & $\begin{array}{ll}0,21 & c \\
0,29 & b c \\
0,35 & b \\
0,61 & a\end{array}$ & $\begin{array}{l}s / d \\
s / d \\
s / d \\
s / d\end{array}$ & $\begin{array}{l}s / d \\
s / d \\
1,66 \\
s / d\end{array}$ \\
\hline 1994 & $\begin{array}{l}\text { Met. FAO } \\
120 \mathrm{l} / \text { día } \\
80 \mathrm{l} / \mathrm{dia} \\
\text { Secano }\end{array}$ & $\begin{array}{ll}282 & b \\
336 & b \\
418 & b \\
583 & a\end{array}$ & $\begin{array}{ll}0,22 & c \\
0,27 & c \\
0,33 & b \\
0,51 & a\end{array}$ & $\begin{array}{ll}1,1 & c \\
1,2 & c \\
1,6 & b \\
2,6 & a\end{array}$ & $\begin{array}{l}49,7 \text { a } \\
60,8 \text { a } \\
67,9 \text { a } \\
59,1 \text { a }\end{array}$ \\
\hline 1995 & $\begin{array}{l}\text { Met. FAO } \\
120 \mathrm{l} / \text { día } \\
80 \mathrm{l} / \text { día } \\
\text { Secano }\end{array}$ & $\begin{array}{rl}94 & d \\
203 & c \\
274 & b \\
534 & a\end{array}$ & $\begin{array}{ll}0,08 & c \\
0,15 & b c \\
0,22 & b \\
0,49 & a\end{array}$ & $\begin{array}{ll}0 & d \\
1,3 & c \\
1,7 & b \\
3,1 & a\end{array}$ & $\begin{array}{l}20,1 \text { d } \\
28,5 \text { c } \\
36,5 \text { b } \\
46,1 \text { a }\end{array}$ \\
\hline
\end{tabular}

(1) 0: ausencia total; 1: casi imperceptible; 2: ligero; 3: medio; 4: grande En cada año, los valores de cada columna seguidos por letras diferentes difieren significativamente al nivel $p \leq 0,05$ según el test de la M.D.S.

El amargor (Tabla IV) es uno de los atributos que caracterizan a los aceites de la variedad Picual. Hemos caracterizado el grado de amargor de los acei- tes empleando el $K_{225}$, teniendo en cuenta la alta correlación existente entre este parámetro y el índice de amargor sensorial estimado por el panel analítico (Gutiérrez Rosales et al., 1992). En los tres años se han observado diferencias significativas entre los valores del $K_{225}$ de los aceites de secano (más amargos) y los de regadío (más dulces), observándose igualmente diferencias significativas entre las diferentes dosis de riego, siendo los aceites procedentes de los olivos mejor regados los que presentaron un $K_{225}$ más bajo, y por tanto un menor grado de amargor. Es este uno de los aspectos prácticos más interesantes de este trabajo, por lo que de confirmarse estos resultados en otros olivares en los próximos años, podría recomendarse la separación de frutos de secano y riego en el momento de recepción en la almazara, posibilitando también en regadío adelantar las fechas de recolección con la finalidad de obtener aceites poco amargos y más frutados.

El contenido total en polifenoles, es un parámetro que está relacionado con el amargor y con la estabilidad de los aceites (Gutiérrez González-Quijano et al., 1977; Tous y Romero, 1993; Uceda y Hermoso, 1994). La figura 3, que presenta la relación existente entre el contenido en polifenoles y el $K_{225}$ para todos los valores obtenidos en los años 1994 y 1995 en los cuatro tratamientos comparados, muestra una altísima correlación positiva $\left(R^{2}=0.96\right)$ entre ambos parámetros, diferenciándose una región correspondiente al aceite de riego y otra correspondiente al de secano.

En la tabla IV se observa igualmente cómo el contenido en polifenoles, $K_{225}$ y valoración sensorial del amargor (evaluada por el panel analítico) están muy relacionadas entre sí.

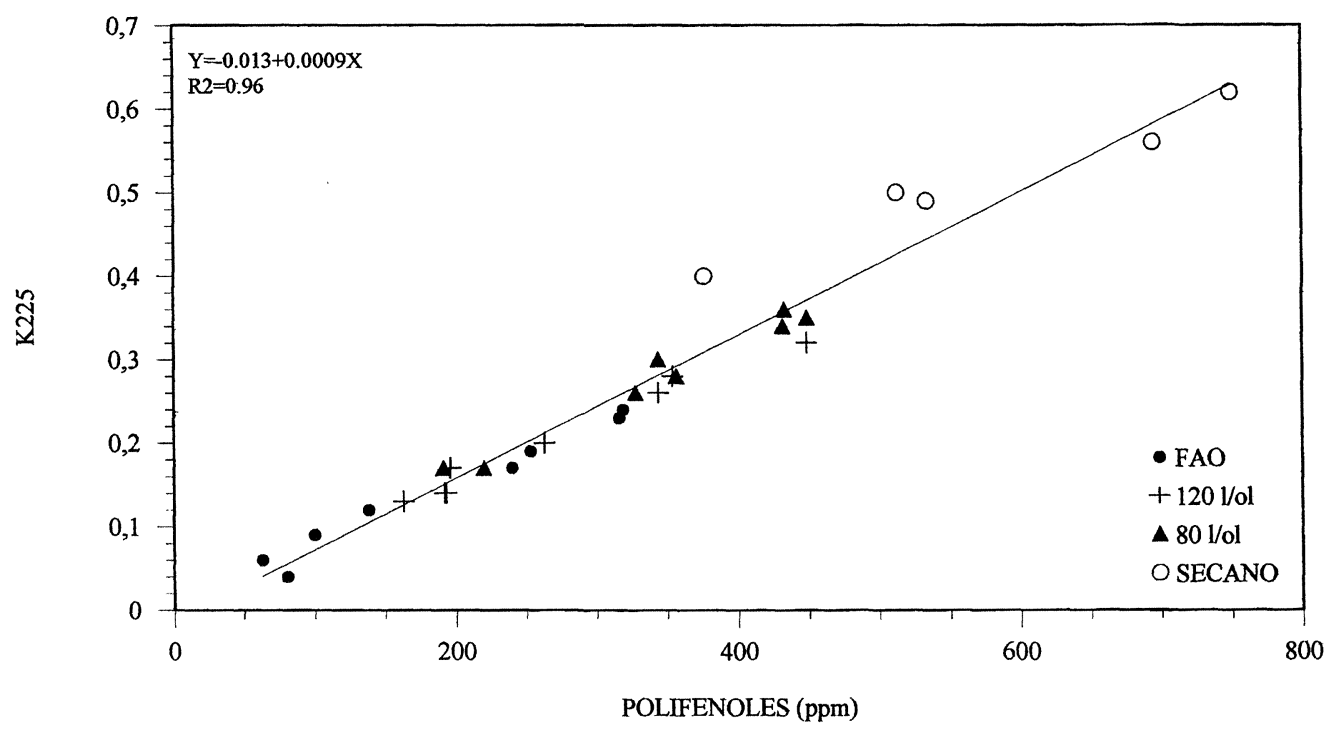

Figura 3

Relación existente entre el contenido total en polifenoles y el k225 en aceites producidos en los diferentes tratamientos de riego en los años 1994 y 1995 
La valoración organoléptica de los aceites producidos en 1994 y 1995 (Tabla V) muestra en general una puntuación alta para todos los tratamientos, aunque esta puntuación fue mayor en el aceite producido en secano. Debemos advertir que se trata de la valoración del panel analítico y no de la valoración por un posible panel de consumidores, en especial si consideramos que el amargor es un atributo que puede no agradar a consumidores no habituales. Para este atributo todos los catadores del panel fueron capaces de distinguir en ambos años los aceites de riego de los de secano, mucho más amargos estos últimos, mientras que el $77 \%$ de los catadores identificó como diferente el aceite producido por los olivos regados con la mayor dosis de agua (Met. FAO) con respecto al producido bajo regímenes de riego deficitario (80 y 120 l/día). Los atributos frutado y picante fueron igualmente afectados por el riego, siendo más patentes estos atributos en los aceites procedentes del cultivo en secano (Tabla V). Hay que destacar la peor calidad organoléptica obtenida en el año 1995 en los aceites producidos por los olivos mejor regados (Met. FAO), obteniéndose una puntuación media igual a 5,4 (aceites con frutado casi imperceptible y amargor nulo), que fue muy inferior a la puntuación obtenida por los restantes tratamientos, lo que podría explicarse porque, tal como se dijo anteriormente, en el momento de la recolección parte de las aceitunas de este tratamiento ya habían caído al suelo de forma natural.

\section{Tabla V}

Atributos sensoriales y valoración organoléptica de los aceites producidos en los años 1994 y 1995 (Los valores representan la media \pm error estándar)

\begin{tabular}{cccccc}
\hline AÑOS & $\begin{array}{c}\text { TRATAMIENTOS } \\
\text { DE RIEGO }\end{array}$ & Amargo & $\begin{array}{c}\text { Atributos (1) } \\
\text { Picante }\end{array}$ & Frutado & $\begin{array}{c}\text { Valoración } \\
\text { organoléptica (2) }\end{array}$ \\
\hline 1994 & Met. FAO & $1.1 \pm 0,23$ & $1.4 \pm 0.23$ & $1.8 \pm 0.18$ & $7.0 \pm 0.22$ \\
& 120 l/día & $1.2 \pm 0.21$ & $1.7 \pm 0.25$ & $1.5 \pm 0.15$ & $6.7 \pm 0.20$ \\
& 80 I/día & $1.6 \pm 0.24$ & $1.8 \pm 0.22$ & $1.9 \pm 0.15$ & $7.2 \pm 0.18$ \\
& Secano & $2.6 \pm 0.14$ & $2.3 \pm 0.33$ & $2.9 \pm 0.15$ & $7.9 \pm 0.07$ \\
1995 & Met. FAO & 0.0 & s/d & $0.5 \pm 0.10$ & $5.4 \pm 0.16$ \\
& 120 l/día & $1.3 \pm 0.15$ & s/d & $2.0 \pm 0.10$ & $7.3 \pm 0.15$ \\
& 80 I/día & $1.7 \pm 0.18$ & s/d & $2.1 \pm 0.20$ & $7.4 \pm 0.17$ \\
& Secano & $3.1 \pm 0.11$ & s/d & $3.0 \pm 0.25$ & $7.8 \pm 0.09$ \\
\hline
\end{tabular}

(1) 0: ausencia total; 1: casi imperceptible; 2: ligero; 3: medio; 4: grande

(2) Escala comprendida entre 1 (peor calidad) y 9 (máxima calidad)

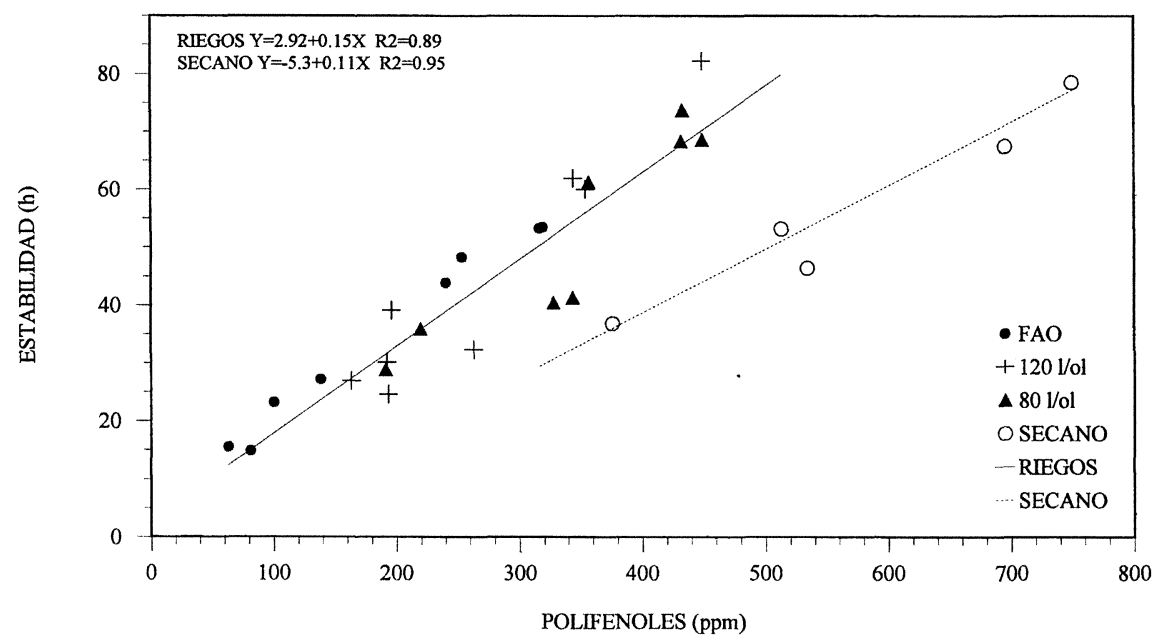

Figura 4

Relación existente entre el contenido total en polifenoles y la estabilidad en aceites producidos en los diferentes tratamientos de riego en los años 1994 y 1995. Se presentan dos ajustes para aceites de secano y para los distintos tratamientos de riego. 
La estabilidad oxidativa medida en Rancimat es un parámetro que proporciona una buena estimación de la susceptibilidad del aceite a la degeneración auto oxidativa, que en los aceites de oliva conduce fundamentalmente a su enranciamiento. En 1995 (Tabla IV) la estabilidad del aceite decreció significativamente al aumentar la dosis de riego aplicada, tendencia que ya se observó en 1994 en los aceites procedentes de los árboles mejor regados.

En la Figura 4 mostramos igualmente la alta correlación existente entre el contenido total en polifenoles y la estabilidad de los aceites (años 1994 y 1995), observándose una pauta de comportamiento diferente para los aceites de riego y los de secano, lo que podría explicarse por diferencias no sólo cuantitativas, sino probablemente también por diferencias cualitativas en el contenido en polifenoles de los aceites, hipótesis que debe ser comprobada en próximos trabajos de investigación.

\section{DISCUSIÓN}

Del conjunto de resultados obtenidos, que deben ser considerados como preliminares, se concluye que existen cambios en la composición química y organolépticas de los aceites producidos en olivos sometidos mediante riego (Figura 1) a diferentes disponibilidades de agua a lo largo de su ciclo vegetativo, lo que determina que en algunos tratamientos los olivos hayan sufrido un fuerte estrés hídrico (Figura 2) durante el período de crecimiento del fruto (verano y otoño), hasta el momento de producirse las primeras lluvias otoñales.

Aunque se han detectado diferencias significativas en determinadas propiedades químicas de los aceites producidos en árboles sometidos a diferentes disponibilidades de agua, en ocasiones estos cambios tienen escasa relevancia desde el punto de vista práctico. Es el caso de la composición acídica, $K_{270}, K_{232}$, acidez e índice de peróxidos, que casi siempre estuvieron dentro de los límites reglamentos (COI, 1992).

El cambio más relevante observado es la variación en el contenido total en polifenoles del aceite en función de las disponibilidades de agua por el olivo (Tabla IV). El contenido en polifenoles está muy correlacionado con el amargor (Figura 3) y con la estabilidad oxidativa de los aceites (Figura 4), lo que sí puede tener una repercusión práctica importante a la hora de tomar decisiones sobre la fecha de recolección y sobre el manejo de los frutos en la almazara, en especial en zonas en las que la superficie de olivar regado tiene un peso específico muy importante dentro del olivar de la comarca. Tengamos en cuenta que en Andalucía una alta proporción de los aceites se extraen en Cooperativas, cuyas almanzaras tienen una gran capacidad diaria de molturación y poseen diferentes líneas de extracción, lo que permitiría una separación de frutos de riego y secano en el momento de su recepción.

Aunque no se han encontrado en la literatura especializada referencias que en el cultivo del olivar relacionen el estrés hídrico sufrido por las plantas con el contenido total en polifenoles del aceite, sin embargo, en otras especies se ha descrito cómo el estrés hídrico puede modificar el contenido de polifenoles en la planta (Rhodes, 1985; Alonso et al., 1993), lo que puede estar en consonancia con nuestros resultados, al haberse comprobado que en los árboles que sufrieron un mayor estrés hídrico fue en los que el contenido total en polifenoles del aceite fue mayor.

\section{AGRADECIMIENTOS}

Agradecemos al Laboratorio Agroalimentario de Córdoba y a la Estación Experimental de Olivicultura "Venta del Llano» (Mengíbar-Jaén) por su valiosa colaboración en la realización de los análisis de aceite. Asimismo agradecemos a D. José M. ${ }^{a}$ Pastor Bueno de Villacarrillo, que cedió desinteresadamente la parcela de olivar en la que se realizaron los ensayos, La R.A.E.A. de la Consejería de Agricultura y Pesca de la Junta de Andalucía financió el ensayo de campo.

\section{BIBLIOGRAFÍA}

Alonso, M. Bara, S. Vega, J. A. (1993). ««Ácidos fenólicos como parámetros bioquímicos indicadores de stress en viveros de Pinus pinaster».- Invest. Agrar., Sist. Recur. For. 2 (2), 185-196.

Beltrán, G., Jiménez, A., Uceda, M. (1995). -«Efecto del Régimen Hídrico de Cultivo sobre la Fracción Fenólica del Aceite de Oliva de la Variedad Arbequina».-Actas del $1^{\text {er }}$ Simposio del Olivo Arbequina en Cataluña. Borjas Blancas. 153-155.

C.O.I. (1992). - «Norma Internacional aplicable a los aceites de oliva y a los aceites de orujo de aceituna".- OLIVAE 40 (2), 44-45.

Civantos, L., Contreras, R., Grana, R. M. (1992). - Obtención del aceite de oliva virgen».- Ed. Agrícola Española, S. A. 47-56.

Dettori, S. (1987). - «Estimación con los métodos de la FAO de las necesidades de riego de los cultivos de aceitunas de mesa en Cerdeña».- OLIVAE 17, 30-35.

Dettori, S., Russo, G. (1993). - «Influencia del cultivar y del régimen hídrico sobre el volumen y la calidad del aceite de oliva».- OLIVAE 49, 36-43.

Doorembos, J., Pruitt, W. O. (1997). - «Las necesidades de agua de los cultivos».- Estudio F.A.O. Riego y Drenaje no 24. Roma.

Frías, L., García-Ortiz, A., Hermoso, M., Jiménez, A., Llavero, M. P., Morales, J., Ruano, M. T., Uceda, M. (1991). - «Analistas de laboratorio de almazara».- Junta de Andalucía. Consejería de Agricultura y Pesca. Serie Apuntes, 6/91. 
Goldhamer, D. A., Dunai, J., Ferguson, L. F. (1994)."Irrigation requirements of olive trees and responses to sustained deficit irrigation". - Acta Horticuturae 356, 172175.

Gutiérrez González-Quijano, R., Janer del Valle, C. Janer del Valle, M. L., Gutiérrez Rosales, F., Vázquez Roncero, A. (1977). -«Relación entre los polifenoles y la calidad y estabilidad del aceite de oliva virgen".- Grasas y Aceites 28 (2), 101-106.

Gutiérrez Rosales, F., Perdiguero, S., Gutiérrez, R., Olías, J. M. (1992). - «Evaluation of the Bitter Taste in Virgin Olive Oil».- J. Am. Oil Chemists'Soc. 69 (4), 394-395.

Inglese, P., Barone, E., Gullo, G. (1996). -«The effect of complementary irrigation on fruit growth, ripening pattern and oil characteristics of olive (Olea europea L.) cv. Carolea». -Journal of Horticultural Science 71 (2), 257263.

Michelakis, N. (1995). - «Efecto de las disponibilidades de agua sobre el crecimiento y el rendimiento de los olivos". - OLIVAE 56, 29-39.

Pastor, M., Castro, J., Manrique, T., Gómez Beltrán, L. (1996). - «Crecimiento del fruto, formación de aceite y producción del olivar regado con diferentes programas de riego por goteo en Andalucía".- Fruticultura Profesional 76, 6-23.

Pastor, M., Orgaz, F. (1994). -«Riego deficitario del olivar».Agricultura 746, 768-776.

Rotundo, A., Ruggiero, C., D'Antonio, P. (1993). -«Effetti dell'irrigazione sullo stato idrico della pianta, sullo sviluppo vegetativo e sulla produzione dell'olivo (Cv. Coratina)".- L'Informatore Agrario 18, 93-99.

Tous, J., Romero, A. (1993). - «Variedades de Olivo. Con especial referencia a Cataluña».- Ed. Fundación La Caixa. Barcelona. 172. pp.

Uceda, M., Hermoso, M. (1994). -«Aceites andaluces. Denominaciones de Origen».- Olivicultura. Jornadas Técnicas. Ed. Fundación La Caixa-Editorial Agro Latino, S. L., Barcelona. 113-120. 